\title{
Prenatal Exposure to the Viral Mimetic Poly I:C Alters Fetal Brain Cytokine Expression and Postnatal Behaviour
}

\author{
Udani Ratnayake ${ }^{a, c}$ Tracey Quinn ${ }^{a}$ Domenic A. LaRosa ${ }^{a}$ Hayley Dickinson ${ }^{a}$ \\ David W. Walker ${ }^{a, b}$ \\ ${ }^{a}$ Ritchie Centre, Monash Institute of Medical Research, Monash University, and b Department of Obstetrics and \\ Gynaecology, Monash University, Clayton, Vic., and 'The Florey Institute of Neuroscience and Mental Health, \\ University of Melbourne, Parkville, Vic., Australia
}

\author{
Key Words \\ Cytokines · Behaviour · Poly I:C · Prenatal exposure · Spiny \\ mouse $\cdot$ Mental illness
}

\begin{abstract}
An increased incidence of mental illness disorders is found in children and adolescents born to mothers who experienced an infection-based illness during pregnancy. Animal models to study the prenatal origin of such outcomes of pregnancy have largely used conventional rodents, which are immature (altricial) at birth compared with the human neonate. In this study, we used the precocial spiny mouse (Acomys cahirinus), whose offspring have completed organogenesis at birth, and administered a single subcutaneous injection of a $5 \mathrm{mg} / \mathrm{kg}$ dose of the viral mimetic poly I:C (polyriboinosinic-polyribocytidylic acid) at mid gestation (20 days; term is 39 days). Prenatal exposure to poly l:C caused a transient weight loss in the pregnant dam, produced a downregulation of the proinflammatory cytokine tumour necrosis factor-a in the fetal brain, and resulted in abnormalities in sensorimotor gating and reduced social interaction, memory and learning in juvenile offspring. No changes in exploratory activity or anxiety and fear behaviours were

found between the treatment groups. This study provides
\end{abstract}

\section{KARGER}

E-Mail karger@karger.com

www.karger.com/dne evidence that, in a rodent model that more closely resembles human brain development, prenatal infection can lead to behavioural abnormalities in postnatal life.

(c) 2014 S. Karger AG, Base

\section{Introduction}

While the aetiology of mental illnesses such as schizophrenia and autism remains unknown, many epidemiological studies have identified a potential neurodevelopmental origin for these disorders [1-6]. These studies have shown an association between infection during pregnancy and increased risk of the development of mental illness disorders in later life. Given that prenatal infection of varying origin and type each appear able to elicit similar postnatal effects [3-9], it is thought that the specific pathogen is not important, but it is the common inflammatory response that is a key mechanism in provoking an alteration in the trajectory of brain development. As infection and inflammation invariably involve the release of inflammatory cytokines, this is known as the cytokine hypothesis [10-13].

Animal studies have sought to investigate the link between exposure to a prenatal infection and the develop-
(C) 2014 S. Karger AG, Basel

0378-5866/14/0362-0083\$39.50/0
Udani Ratnayake, $\mathrm{BSc}$ (Hons), $\mathrm{PhD}$

Melbourne Brain Centre, University of Melbourne

30 Royal Parade

Melbourne, VIC 3010 (Australia)

E-Mail Udani.Ratnayake@florey.edu.au 
ment of mental illness disorders in postnatal life by using the viral mimetic poly I:C (polyriboinosinic-polyribocytidylic acid). Administration of poly I:C in rodents has been shown to cause an increase in inflammatory cytokines and plasma corticosterone, to induce a sickness behaviour syndrome including a febrile response, and to reduce appetite and decrease body weight [14-16]. Previous studies have described behavioural abnormalities in offspring born to pregnant mice and rats given poly I:C during gestation [17-25], even though brain development in these rodents is much less advanced at birth than it is in human pregnancy. As a model of viral illness appropriate to second-trimester pregnancy in women, we have used the spiny mouse (Acomys cahirinus), an animal whose brain development is more advanced by the time of birth [26]. Indeed, the spiny mouse may be said to be precocial in that organogenesis is largely complete by the time of birth, and the neonates are capable of motor activity and thermoregulation [27-31]. In addition, the spiny mouse secretes cortisol as the major circulating glucocorticoid (cf. corticosterone in rodents), and the fetal adrenal gland also produces dehydroepiandrosterone [32], an important androgen involved in brain development that conventional mice and rats do not produce in utero.

Bacterial and viral infections are recognised by the Toll-like receptors (TLRs) of the innate immune system. Poly I:C binds to and activates the TLR3, a receptor that recognises the double-stranded RNA produced by most viruses during their replication [33]. Recognition of infections by TLRs results in the activation of 'nuclear factor kappa-light-chain-enhancer of activated B cells' (NF$\kappa \mathrm{B})$ transcription factors, resulting in the subsequent production of inflammatory cytokines [34-36] - in particular of interleukin (IL)- 6 and tumour necrosis factor (TNF)- $\alpha$, two cytokines that are constitutively altered in the serum of many schizophrenia and autistic patients [37-40], and in the brain of fetal rats and mice after maternal administration of poly I:C [18, 41-44].

We sought to determine whether poly I:C administered to the precocial spiny mouse at mid pregnancy, at a stage when myelination of the brain [26] and adrenocortical differentiation is just beginning [32], had an impact on cytokine expression in the fetal brain, and whether prenatal exposure to this viral mimetic had effects on postnatal behaviour. In particular, we examined the effects of prenatal poly I:C administration on prepulse inhibition, which, in conventional rodents, has been used as a signature of a behavioural disorder closely associated with schizophrenia in humans [21-23, 25]. Furthermore, the current study aimed to extend our previous findings
[45] by using a higher dose of poly I:C ( $5 \mathrm{mg} / \mathrm{kg})$, which has been consistently shown to cause deficits in prepulse inhibition and other behavioural abnormalities in rats and mice $[17,19,25,46-48]$.

\section{Animals and Methods}

\section{Animals}

This study used spiny mice (Acomys cahirinus) obtained from the breeding colony maintained at Monash Medical Centre. The spiny mice were bred and housed as previously described [49]. Experiments were conducted in accordance with the Australian Code of Practice for the Care and Use of Animals for Scientific Purposes. All procedures had received prior approval from the Monash University Animal Ethics Committee.

\section{Prenatal Treatment}

Pregnant dams at day 20 of gestation (term is at 39 days) received a single subcutaneous injection of either phosphate-buffered saline (PBS; control) or $5 \mathrm{mg} / \mathrm{kg}$ of poly I:C (polyinosinicpolycytidylic acid potassium salt; Sigma Aldrich, Castle Hill, N.S.W., Australia) in a volume of $5 \mu \mathrm{l} / \mathrm{g}$ body weight. Poly I:C was dissolved in PBS solution to yield the required concentration. Developmental events at 20 days of gestation (mid gestation) in the brains of spiny mice compares with that of the human fetus at early-to-mid gestation [26]. All animals were then returned to their home cages immediately after the injection procedures. The animals either were culled for tissue collection 2 or $24 \mathrm{~h}$ after injection when maternal, fetal and placental weights were obtained or the pregnancies were allowed to continue until the dams gave birth naturally, and the behaviour of the offspring was assessed from 20 to 35 days of age. For all behavioural tests a minimum of 6 male and 6 female offspring from each treatment group were assessed.

\section{Behaviour Tests}

Assessment of behaviour of the offspring occurred at 20 and 35 days of age, which is prior to weaning at about 40 days of age. As the offspring were assessed by all behavioural tests, they were ordered from least stressful to most stressful. Based on the documented observations from our colony [50] and by others [51], spiny mice of this postnatal age can be considered juvenile and prepubertal.

Open Field Test. Offspring at 20 days of age were tested in an open field $(50 \times 50 \times 40 \mathrm{~cm} ; \mathrm{L} \times \mathrm{W} \times \mathrm{H})$ environment to assess $(1)$ exploratory activity by measuring the distance travelled, and (2) anxiety-like behaviours by determining the amounts of time spent in the central zone versus the outer zone of the field. The central zone was defined arbitrarily by the area of the open field excluding a $10-\mathrm{cm}$ outer perimeter. The open field test was conducted between the hours of 11:00 and 13:00 h, and each pup under test, with their siblings and mother, was habituated to the room in which the open field apparatus was kept by placing the home cage in the room for at least $1 \mathrm{~h}$ prior to testing. The lighting level was set at $2.8 \mathrm{~lx}$ for all trials. The offspring were placed in the centre of the field at the beginning of each trial, which lasted $10 \mathrm{~min}$. Limelight (Neuroscience Inc., Tokyo, Japan) acquisition software was used to track the movement of the animals throughout the open field trial by identifying the nose, body and tail. Images were captured 
Fig. 1. The 3 chambers and 2 enclosures used in the social interaction test.

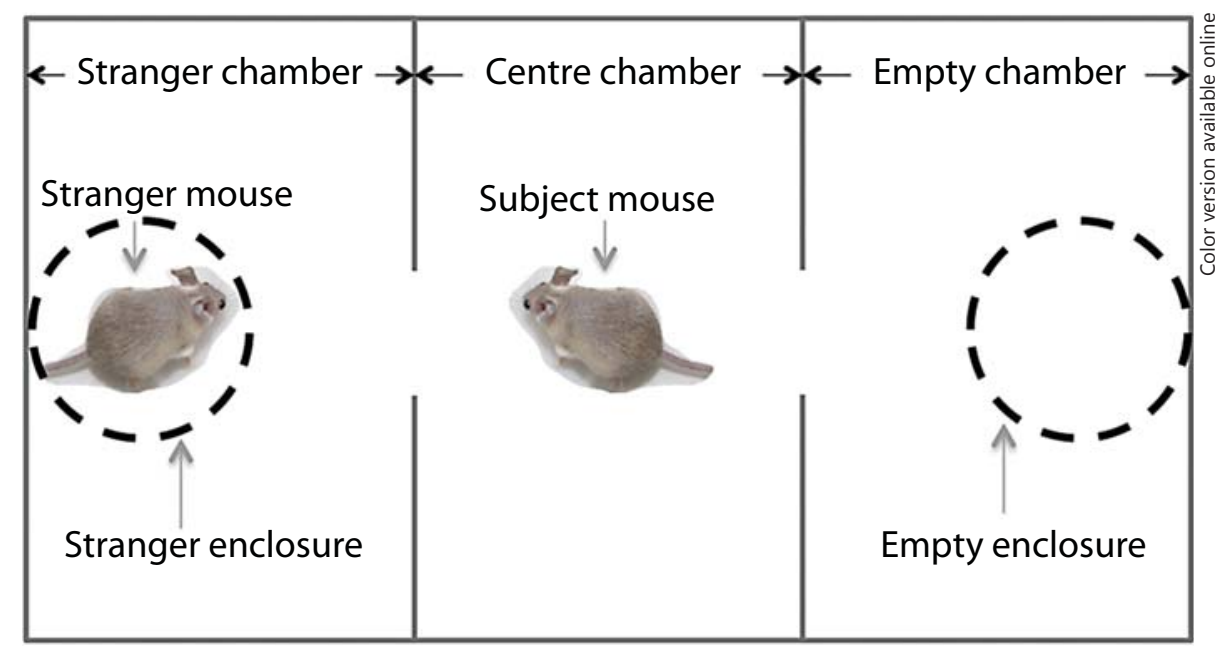

at a rate of $7.5 \mathrm{~Hz}$ and stored on a disc. Postacquisition analysis of the body of the animal using the dedicated software allowed the measurement of distance (in centimetres) travelled throughout the test, and the distance (in centimetres) and time (in seconds) spent in the inner central and outer perimeter zones. The animal was then removed from the open field and returned to the home cage.

Novel Object Recognition Test. Immediately after this open field trial, the novel object recognition test was conducted to assess nonspatial memory, which is based on the innate exploratory behaviour of rodents. The above open field trial was used as the habituation trial for the novel object recognition test to reduce the contribution of the novelty of the open field to the test. Immediately following the completion of the open field test, 2 small plastic blocks of identical shape and size were placed in the open field, positioned $6 \mathrm{~cm}$ from the walls of the arena to ensure an unobscured view of the animal at all times. The animal was then placed back in the open field, and its behaviour recorded for a 10-min period, which served as the learning trial in which the animal acquired information regarding the objects in the open field. Following this, the animal was returned to its home cage for $1 \mathrm{~h}$ (retention period), and during this time 1 of the 2 plastic blocks was replaced with a plastic block of a different shape and colour (the novel object). The remaining plastic block (which had been explored previously in the learning trial) and the novel object were both wiped down with $70 \%$ ethanol to remove olfactory cues. The animal was placed back in the open field, and its behaviour and exploration of the 2 objects was recorded for the next 10 min (termed the 'recall trial'). In the analysis of the behaviour during the learning and recall trials, the nose rather than the body of the animal was tracked using the Limelight software. When the animal's nose was pointed at and within $2 \mathrm{~cm}$ of the block, this was deemed to be active exploration of the block. The total time spent exploring objects in the 10 min of the learning trial was measured. The total time spent exploring each object for the first $5 \mathrm{~min}$ in the recall trial was used to determine the discrimination index, calculated by subtracting the time spent exploring the familiar object from the time spent exploring the novel object, divided by the total time spent exploring both objects. Thus, a discrimination index of 1 indicates that the animal spent all the time exploring the novel object, 0 indicates no preference for either object, and -1 represents all time spent exploring the familiar object.

Social Interaction Test. The social interaction test was used to assess whether the poly I:C treatment had caused changes in the social behaviours of the offspring at 25 days of age. The apparatus consisted of an open top rectangular box of $60 \times 30 \times 45 \mathrm{~cm}(\mathrm{~L} \times$ $\mathrm{W} \times \mathrm{H})$ divided into 3 chambers of $20 \times 30 \mathrm{~cm}(\mathrm{~L} \times \mathrm{W})$, with an $8 \times 8 \mathrm{~cm}$ square opening cut into the internal walls to allow the animal to move between chambers. The chambers were numbered 1-3 from left to right for reference. Chambers 1 and 3 contained large cylinders measuring $40 \times 8 \mathrm{~cm}(\mathrm{H} \times \mathrm{D})$ drilled with numerous $0.6-\mathrm{cm}(\mathrm{D})$ holes, in 1 of which the 'stranger' spiny mouse was placed during the test. The stranger spiny mouse was age and sex matched to the test spiny mouse. The social interaction tests consisted of the test spiny mouse being habituated to the social interaction apparatus for a 5-min period, after which the spiny mouse was taken out and the stranger spiny mouse placed in the cylinder enclosure in chamber 1 or 3 . The placement of the stranger spiny mouse in the enclosure was alternated between chambers 1 and 3 for animals in each treatment group to account for preference to a particular chamber of the apparatus. The test spiny mouse was placed back in the middle of chamber 2 and a video recording was taken as the spiny mouse explored the apparatus. The stranger spiny mouse was available to the test spiny mouse for visual, tactile and olfactory contact through the enclosure. Postacquisition analysis of the time spent in the stranger, centre or empty chambers was performed and the time spent interacting with the stranger or empty enclosure by the subject spiny mouse was measured using the Limelight software. The chamber and enclosure definitions are shown in figure 1 . When the animal's nose was pointed at and within $2 \mathrm{~cm}$ of the stranger spiny mouse's enclosure, this was deemed to be interaction with the stranger spiny mouse.

Elevated Plus Maze. Animals at 30 days of age were placed on the elevated plus maze apparatus to assess anxiety and fear behaviour in a novel and challenging environment. The elevated plus maze test was conducted between 11:00 and 13:00 h. The elevated plus maze consisted of 2 opposing open arms $(40 \times 10 \mathrm{~cm})$ and 2 opposing closed arms (also $40 \times 10 \mathrm{~cm}$ ) which are surrounded by 15-cm-high walls. The 4 arms were connected in the shape of a 
Table 1. Primer sequences for genomic analysis

\begin{tabular}{lll}
\hline & Forward primer & Reverse primer \\
\hline $\begin{array}{ll}\text { Housekeeping gene RN18S1 } \\
(\text { RNA, 18S ribosomal 1) }\end{array}$ & ACACGGACAGGATTGACAGA & CAAATCGCTCCACCACCAACTAA \\
NF- $\mathrm{B} B$ & TGAGGGATCTGCTGGAAGTC & CCAAGTGCAGAGGTTCTGA \\
IL-6 & CAGAGCCATTCAGAGCAACA & TGACCTGGATCCTTCACCTC \\
TNF- $\alpha$ & CAAATTCGAGTGACAAGCCTG & GAGATCCATCCCGTTGGC \\
\hline
\end{tabular}

Table 2. Effect of prenatal PBS and poly I:C administration on sickness behaviours and pregnancy outcomes

\begin{tabular}{|c|c|c|c|}
\hline & PBS & Poly I:C & $\mathrm{p}$ value \\
\hline \multicolumn{4}{|c|}{ Maternal, fetal and placental data } \\
\hline \multicolumn{4}{|c|}{ Maternal body weight } \\
\hline change, $g$ & $0.06 \pm 0.70$ & $-1.78 \pm 0.26^{\mathrm{b}}$ & $0.04^{\mathrm{a}}$ \\
\hline Fetal body weight, g & $0.49 \pm 0.05$ & $0.29 \pm 0.03$ & $0.01^{\mathrm{a}}$ \\
\hline Placental weight, g & $0.23 \pm 0.02$ & $0.14 \pm 0.01$ & $0.004^{\mathrm{a}}$ \\
\hline \multicolumn{4}{|l|}{ Placental:fetal body weight } \\
\hline ratio & $0.50 \pm 0.07$ & $0.51 \pm 0.07$ & 0.87 \\
\hline \multicolumn{4}{|l|}{ Pregnancy outcomes } \\
\hline Gestational period, days & $39.00 \pm 0.21$ & $38.88 \pm 0.23$ & 0.70 \\
\hline Litter size, $\mathrm{n}$ & $2.75 \pm 0.48$ & $3.25 \pm 0.48$ & 0.49 \\
\hline
\end{tabular}

cross to a central platform $(10 \times 10 \mathrm{~cm})$. The entire apparatus was elevated on a stand, hidden to the animal's view, $50 \mathrm{~cm}$ above the floor. The test began when the spiny mouse was placed on the central platform of the maze facing an open arm and allowed to move freely for $5 \mathrm{~min}$, during which time its movements were recorded by a video camera placed above the apparatus. Limelight acquisition software was used to track the movement of the animal and assess the distance travelled and time spent in each arm throughout the trial.

Prepulse Inhibition Test. Prepulse inhibition (PPI) of acoustic startle was measured using the SR-LAB startle apparatus (San Diego Instruments, San Diego, Calif., USA) at 35 days of age. The sound-proofed startle chamber contained a clear Plexiglas cylinder resting on a piezoelectric transducer that detected movement of the animal. A computer connected to the apparatus recorded the startle responses and controlled the timing and presentation of the acoustic stimuli. Testing took place between 10:00 and 13:00 h. Each test began with 2 min of apparatus acclimatisation, followed by 5 consecutive pulse-alone trials presented in order to habituate the animal and establish a stable startle response. The animals were then presented with a total of 40 pseudorandom trials consisting of 10 pulse-alone trials, 5 no-stimulus trials (i.e. background noise), and 5 prepulse + startle pulse trials where the amplitude of the prepulse was $72,74,78,82$ or $86 \mathrm{~dB}$ sound pres- sure level (SPL), corresponding to 2, 4, 8 and $16 \mathrm{~dB}$ SPL above the background noise. The session was concluded with 5 consecutive startle pulse-alone trials. The interval between successive trials ranged from 10 to $30 \mathrm{~s}$, with a mean of $20 \mathrm{~s}$. The startle pulse stimulus was a 40 -ms pulse of white noise at $115 \mathrm{~dB}$ SPL, and the prepulses were 20 -ms bursts of white noise delivered $100 \mathrm{~ms}$ before the startle pulse. The average PPI was expressed as the average of percent inhibition of the startle response due to each prepulse, i.e. $\% \mathrm{PPI}=$ amplitude of startle pulse - (amplitude of each prepulse + startle pulse) $/($ amplitude of startle pulse $) \times 100 \%$. A higher $\%$ PPI score indicates a greater reduction in startle magnitude in prepulse + pulse trials relative to that in pulse-alone trials. The average startle was expressed as the average amplitude of the startle pulse-alone trials.

\section{Quantitative Real-Time PCR}

Samples (placenta, fetal) were obtained at $2 \mathrm{~h}(\mathrm{n}=5 \mathrm{dams})$ and $24 \mathrm{~h}$ ( $\mathrm{n}=5$ dams) after poly I:C administration to assess cytokine gene expression. Total RNA was extracted and DNase treated using the commercially available RNeasy Kits (Qiagen, Malvern East, Vic., Australia). SYBR green PCR master mix, as per the manufacturer's instructions (Applied Biosystems), was used to determine relative levels of mRNA expression for $N F-\kappa B, T N F-\alpha$ and $I L-6$. The primer sequences are listed in table 1 . The PCR assay was optimised by determining the specificity of the primer sets, which were found to amplify products of the expected sizes. Quantitative (q)PCR cycling conditions for all genes consisted of an initial denaturation step of $95^{\circ} \mathrm{C}$ for $10 \mathrm{~min}$, followed by 40 cycles consisting of $95^{\circ} \mathrm{C}$ for $15 \mathrm{~s}$ and $60^{\circ} \mathrm{C}$ for $1 \mathrm{~min}$. A dissociation curve was also performed at the end of each run. Samples were run in triplicate for each gene of interest. Data obtained from qPCR were analysed as previously described [52]. The relative expression within the sample $\left(\Delta \mathrm{C}_{\mathrm{T}}\right)$ was measured from the $\mathrm{qPCR}$ data by subtracting the mean cycle threshold $\left(\mathrm{C}_{\mathrm{T}}\right)$ for the housekeeping gene $(18 S)$ from the mean $\mathrm{C}_{\mathrm{T}}$ value for the gene of interest. This value was then inserted into the formula $2^{-\Delta \mathrm{CT}}$ to give a final arbitrary expression value, which was then divided by the mean $2^{-\Delta \mathrm{CT}}$ of the PBS2-hour treatment group to give a final value against which the relative expression for each gene of interest was determined for the other time ( $24 \mathrm{~h}$ ) and the poly I:C group at 2 and $24 \mathrm{~h}$ after treatment.

\section{Statistics}

All data are presented as means \pm SEM. Maternal, fetal and placenta data and pregnancy outcomes were analysed using an independent $t$ test. Open field test results, novel object recognition test results, average startle and average change of PPI (expressed as 
Table 3. Effect of prenatal PBS and poly I:C administration on body weight (g) of offspring at 1, 20 and 40 days of age

\begin{tabular}{|c|c|c|c|c|c|}
\hline & \multicolumn{2}{|l|}{ PBS } & \multicolumn{2}{|l|}{ Poly I:C } & \multirow{2}{*}{$\begin{array}{l}\text { Main effects } \\
\text { (p value) }\end{array}$} \\
\hline & male & female & male & female & \\
\hline At 1 day & $5.82 \pm 0.25$ & $5.6 \pm 0.1$ & $5.36 \pm 0.09$ & $5.44 \pm 0.08$ & $\mathrm{p}_{\text {treatment }}=0.06$ \\
\hline At 20 days & $18.31 \pm 1.37$ & $17.16 \pm 1.3$ & $17.08 \pm 0.91$ & $15.67 \pm 0.68$ & $\mathrm{p}_{\mathrm{sex}}=0.06$ \\
\hline At 40 days & $32.05 \pm 1.37$ & $28.4 \pm 0.6$ & $28.7 \pm 0.74$ & $27.75 \pm 0.81$ & $\mathrm{p}_{\text {treatment } \times \text { sex }}=0.47$ \\
\hline
\end{tabular}

percent) were analysed using a two-way analysis of variance (treatment $\times$ sex). Gene expression results were analysed using a twoway analysis of variance (treatment $\times$ time). A two-way repeatedmeasures analysis of variance was used to analyse postnatal growth (treatment $\times$ sex $\times$ age), social interaction test results (treatment $\times$ sex $\times$ chamber/enclosure) and \%PPI for each prepulse intensity (treatment $\times$ sex $\times$ intensity). $\mathrm{p}<0.05$ was accepted as statistically significant unless otherwise stated; however, a p value of $<0.10$ was considered noteworthy.

\section{Results}

Maternal, Fetal and Placenta Weight, Pregnancy

Outcomes and Postnatal Growth

Maternal body weight fell significantly after the poly $\mathrm{I}$ :C treatment and $24 \mathrm{~h}$ after the treatment was significantly lower than the maternal weight of the PBS-treated dams $\left(\mathrm{t}_{8}=2.44, \mathrm{p}=0.04\right.$; table 2$)$. There was also a significant reduction in fetal body weight $\left(\mathrm{t}_{13}=3.116, \mathrm{p}=\right.$ $0.01)$ and placental weight $\left(\mathrm{t}_{13}=3.520, \mathrm{p}=0.004\right)$ at $24 \mathrm{~h}$ after poly I:C administration. When placental weight was taken into account, there was no significant difference between treatment groups in placental-to-fetal weight ratio $\left(\mathrm{t}_{14}=1.451, \mathrm{p}=0.17\right.$; table 2$)$. There was no effect of treatment on litter size or the length of the duration of gestation (table 2). Body weight increased in all offspring between 1 day and 40 days of age (main time effect $F_{2,48}=$ 1,292 , $\mathrm{p}<0.0001$; table 3 ), with female offspring being near-significantly lighter than their male littermates (main sex effect $\mathrm{F}_{1,24}=3.98, \mathrm{p}=0.06$ ). There was also a tendency for poly I:C animals to be lighter throughout postnatal life compared with PBS controls, although this did not quite reach significance (main treatment effect $\left.\mathrm{F}_{1,24}=4.01, \mathrm{p}=0.06\right)$.

\section{Gene Expression}

The time after treatment, or administration of poly I:C, had no effect on NF- $\kappa \mathrm{B}$, IL- 6 and TNF- $\alpha$ mRNA expression in the placenta (fig. 2a, c, e). Similarly, there was no effect on IL-6 mRNA expression in the fetal brain. However, there was a significant decrease in NF- $\kappa B\left(F_{1,15}=6.03, p=\right.$ 0.03; fig. 2b) and TNF- $a\left(F_{1,16}=6.33, p=0.02\right.$; fig. $\left.2 f\right)$ expression in the fetal brain of poly I:C-treated animals compared with the PBS controls; a similar decrease occurred at both 2 and $24 \mathrm{~h}$ after administration of poly I:C.

\section{Open Field Test}

The total distance travelled by offspring was not different between the treatment groups, but there was a strong tendency for female offspring to travel less in the open field trial than males (main sex effect $\mathrm{F}_{1,23}=3.70, \mathrm{p}=0.06$; fig. 3a). There was no effect of prenatal treatment or sex on the distance travelled by offspring in the central zone of the open field (fig. 3b, c).

\section{Novel Object Recognition Test}

There was no difference between the treatment groups in the time spent exploring objects in the initial learning trial (fig. 4a). However, in the second trial, offspring from poly I:C-treated mothers spent less time exploring the novel object than pups from PBS-treated mothers, as shown by the significantly lower discrimination index $\left(\mathrm{F}_{1,23}=9.829, \mathrm{p}<0.01\right.$; fig. $\left.4 \mathrm{~b}\right)$. The results were similar for male and female offspring.

\section{Social Interaction Test}

All offspring recognised the presence of the 'stranger', as shown in figure $5 \mathrm{~b}$, by the significantly greater amount of time spent exploring the stranger spiny mouse's chamber compared with the centre or empty chamber (main effect of chamber $\mathrm{F}_{2,46}=43.795, \mathrm{p}<0.001$ ). However, unlike all other treatment groups, male offspring from the poly I:C mothers did not interact significantly more with the 'stranger' enclosure than with the empty enclosure, so that a significant 'stranger $\times$ treatment $\times$ sex' interaction term was identified $\left(\mathrm{F}_{1,23}=5.184, \mathrm{p}<0.05\right.$; fig. $\left.5 \mathrm{a}\right)$. 

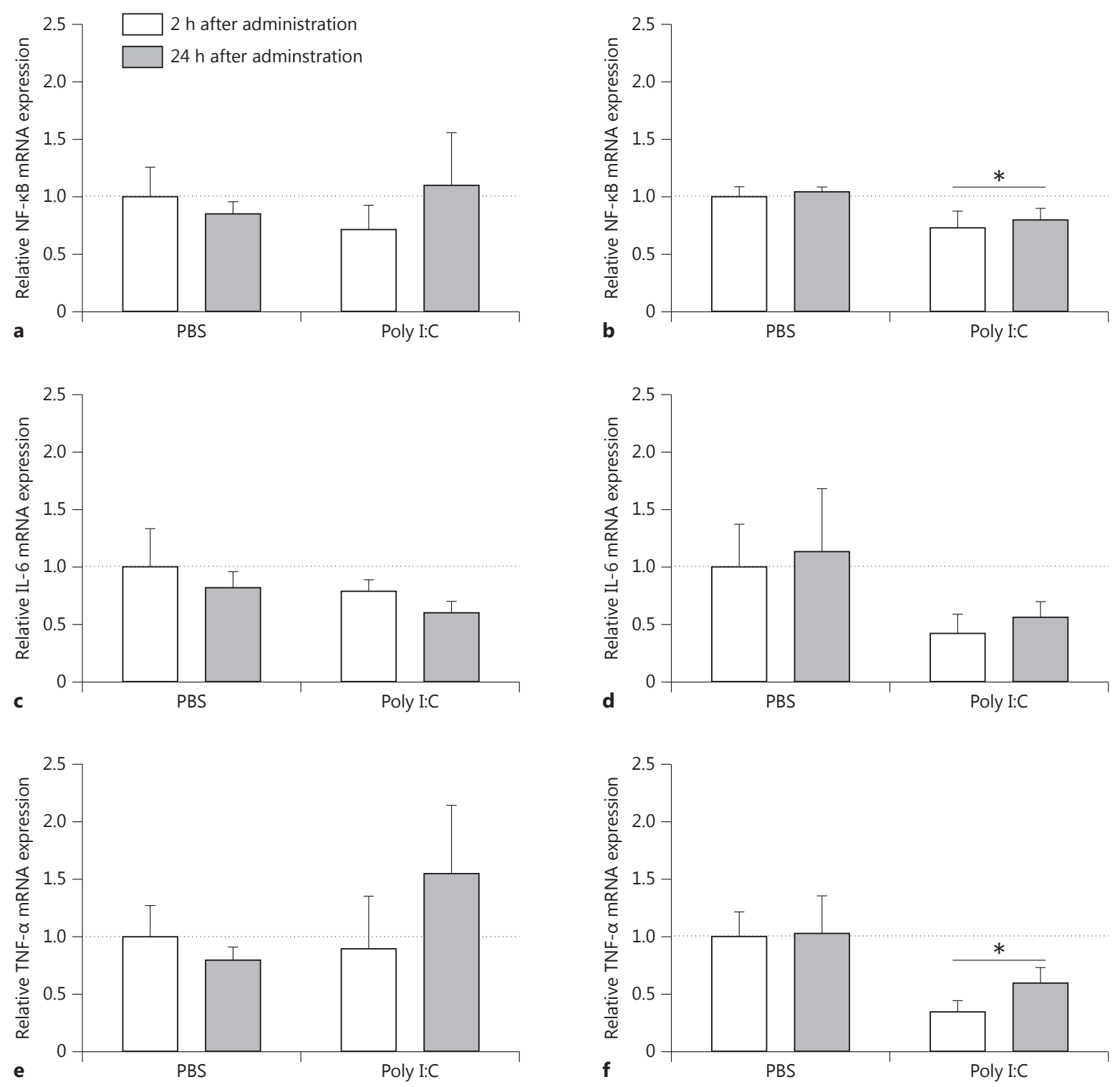

Fig. 2. Relative expression of NF- $\kappa B(\mathbf{a}, \mathbf{b}), \operatorname{IL}-6(\mathbf{c}, \mathbf{d})$ and TNF- $\alpha(\mathbf{e}, \mathbf{f})$ in the placenta $(\mathbf{a}, \mathbf{c}, \mathbf{e})$ and fetal brain $(\mathbf{b}$, d, f) $2 \mathrm{~h}$ and $24 \mathrm{~h}$ after PBS and poly I:C treatment. There was a significant reduction in NF- $\mathrm{kB}(\mathbf{b})$ and TNF- $\alpha$ (f) expression in the fetal brains at 2 and $24 \mathrm{~h}$ after poly I:C treatment compared with PBS. * Main treatment effect of $\mathrm{p}<0.05$. The data are shown as means \pm SEM in all graphs.

\section{Elevated Plus Maze}

Prenatal treatment had no effect on the distance travelled or time spent in the open or closed arms of the elevated plus maze (data not shown).

\section{Prepulse Inhibition}

Average startle was lower in female mice than in their male littermates (main sex effect $\mathrm{F}_{1,21}=6.275, \mathrm{p}<0.05$ ), and the difference between the sexes was similar for the PBS- and poly I:C-treated offspring (fig. 6a). Prenatal treatment with poly I:C significantly reduced \%PPI for each prepulse intensity, and also for the average relative decrease in PPI in these offspring, compared with PBS treatment (main treatment effect $\mathrm{F}_{1,21}=8.023, \mathrm{p}<0.01$ ); a similar effect occurred for males and females (fig. 6b, c). 


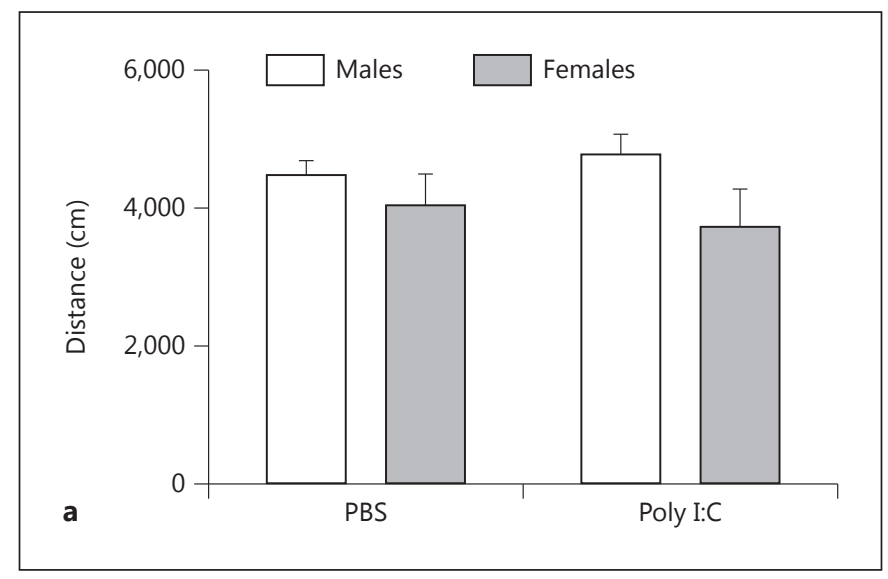

Fig. 3. Total distance travelled (a) and the distance travelled (b) and time spent in the central zone (c) of the open field by male (white columns) and female (dashed columns) offspring prenatally exposed to PBS or poly I:C. There was no main effect of treatment on distance travelled overall and the distance travelled in the central zone of the open field. The data are shown as means \pm SEM in all graphs.

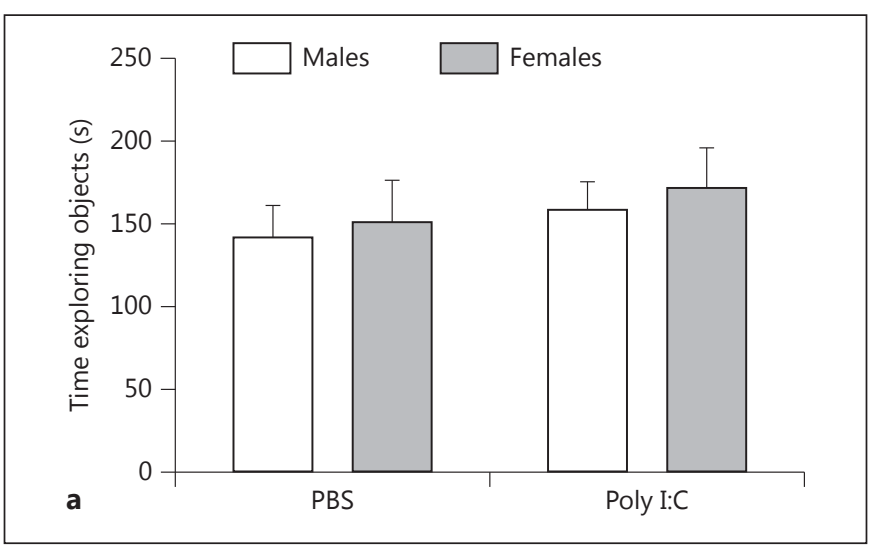

Fig. 4. Time spent exploring objects in the learning period (a) and discrimination index measured in the recall trial (b) of the novel object recognition test by male (white columns) and female (dashed columns) offspring prenatally exposed to PBS or poly I:C.
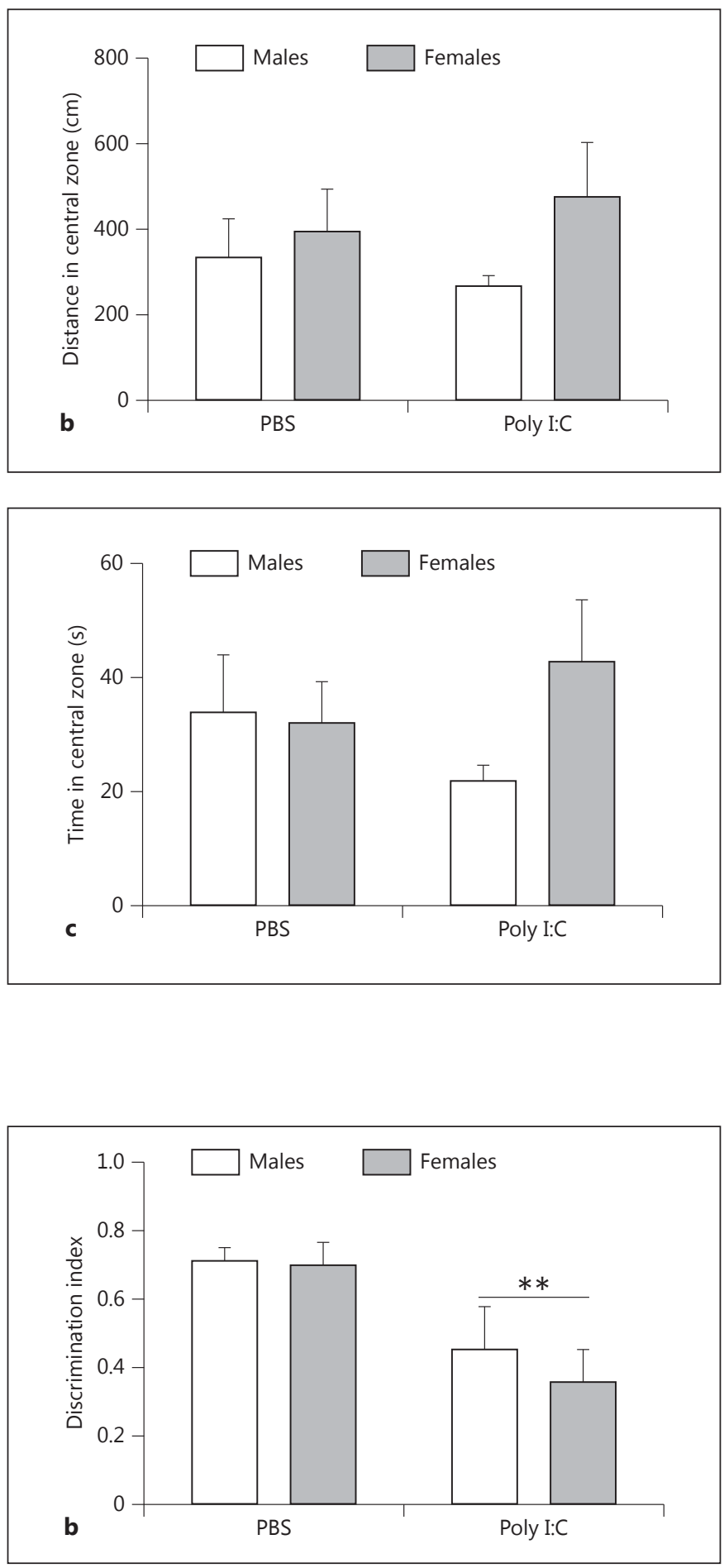

Poly I:C animals had a significantly lower discrimination index than PBS animals $(\mathrm{p}<0.01)$, irrespective of sex. ${ }^{* *}$ Main treatment effect of $\mathrm{p}<0.01$ compared with PBS. The data are shown as means \pm SEM in all graphs. 


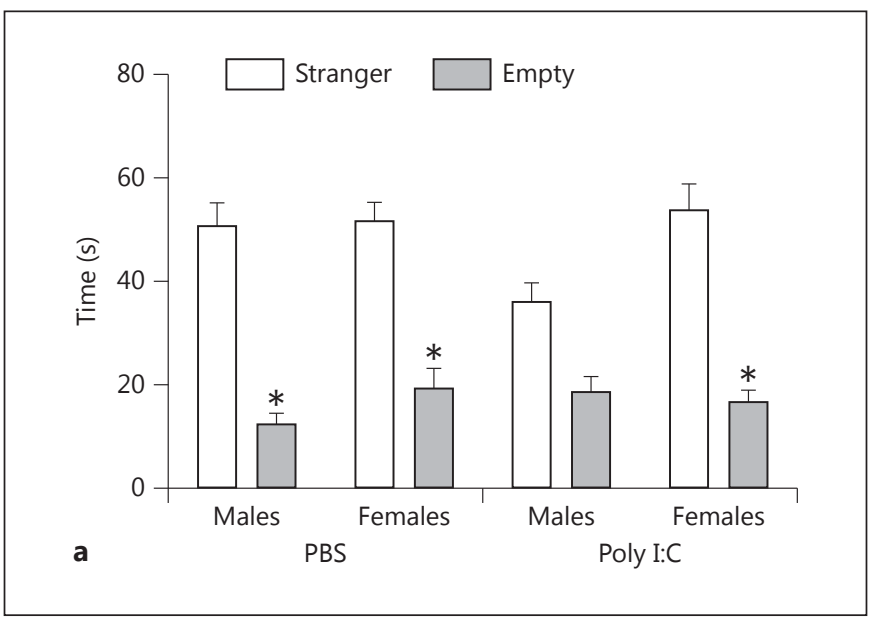

Fig. 5. Time spent interacting with the stranger mouse in the stranger enclosure compared with the empty enclosure (a) and time spent in each chamber (b) by male and female offspring prenatally exposed to PBS or poly I:C. All offspring spent significantly more time exploring the stranger enclosure than the empty en-

\section{Discussion}

This study shows that a virus-like infection during pregnancy, mimicked by using the TLR3 agonist poly I:C, is associated with an acute decrease in cytokine expression in the fetal brain. Cytokines are important inflammatory mediators induced by infection $[53,54]$. In addition to their important role in the innate immune response, cytokines participate in a wide range of important functions including glial and neuronal development [54, 55], and in pregnancy the placenta is also an additional source of cytokine production [56, 57]. Specifically, in this study using the precocial spiny mouse, poly I:C administration at mid gestation acutely (at $2 \mathrm{~h}$ ) resulted in a significant decrease in mRNA levels of the proinflammatory cytokine TNF- $\alpha$ in the fetal brain of the spiny mouse, and this change was also present $24 \mathrm{~h}$ after treatment. While a shift in the relative production of pro- and anti-inflammatory cytokines might be expected to affect brain development [54], this was an unexpected finding because other studies using altricial rodents have described increased proinflammatory cytokine production following maternal poly I:C treatment. Although it is not known what causes this downregulation, it is known that cytokines produced by the maternal immune system can activate the fetal HPA axis [58], resulting in the release of glucocorticoids which then inhibit the induction of proinflammatory cytokines while stimulating the production of anti-inflammatory cytokines $[59,60]$. This negative

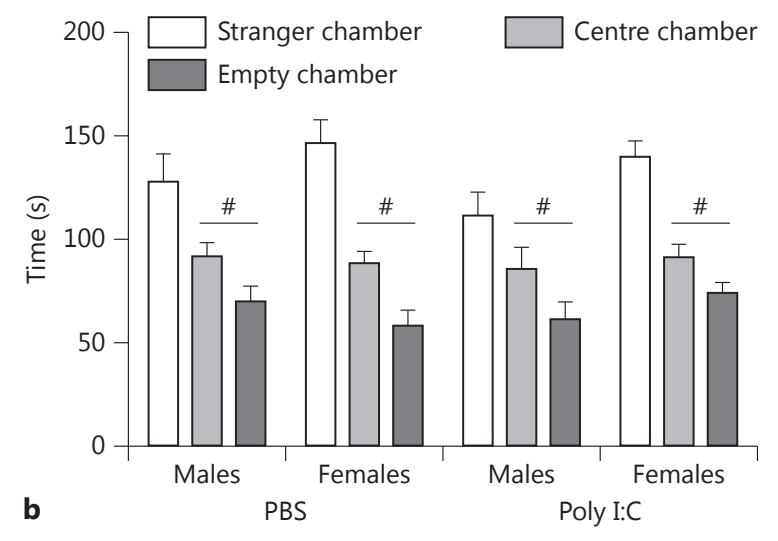

closure, except the male poly I:C animals (a). There was no difference in the time spent in each chamber between the treatment groups or sexes. ${ }^{*} \mathrm{p}<0.05$ compared with stranger enclosure; ${ }^{\#} \mathrm{p}<0.001$ compared with stranger chamber. The data are shown as means $\pm \mathrm{SEM}$ in all graphs.

feedback relationship could explain the downregulation of proinflammatory cytokines in the brain of the fetal spiny mouse shortly after poly I:C treatment.

As the change in cytokine expression was only found in the fetal brain but not the placenta, the question of how this is brought about remains unclear. Cytokines have been found to be present in rodent fetal brains from early gestation $[12,43]$, and in vitro studies demonstrate that cytokines and chemokines can be produced by human fetal microglia and astrocytes in response to viral infection [58, 61-64]. Furthermore, cytokines are thought to be critically important in glial cell development $[54,65]$. This may explain the increase in astrocytes and activated microglia in the neonatal brains of spiny murine offspring exposed to a lower dose of poly I:C at mid gestation, as reported previously [45].

It was found that fetal bodies and placentas were lighter when collected from poly I:C-treated dams, although it is difficult to determine whether either treatment had an effect on the weight of these tissues without knowing the initial weights of the fetuses and placentas. We believe the fetuses were coincidentally smaller in the poly I:C treatment group and that this decrease in placental and fetal weight was not caused by treatment, which is also shown by the fact that the placental-to-fetal weight ratio was not different between the treatment groups.

After birth, prenatal poly I:C treatment resulted in significant changes in the behaviour of the offspring, including deficits in social interaction, sensorimotor gating, and 


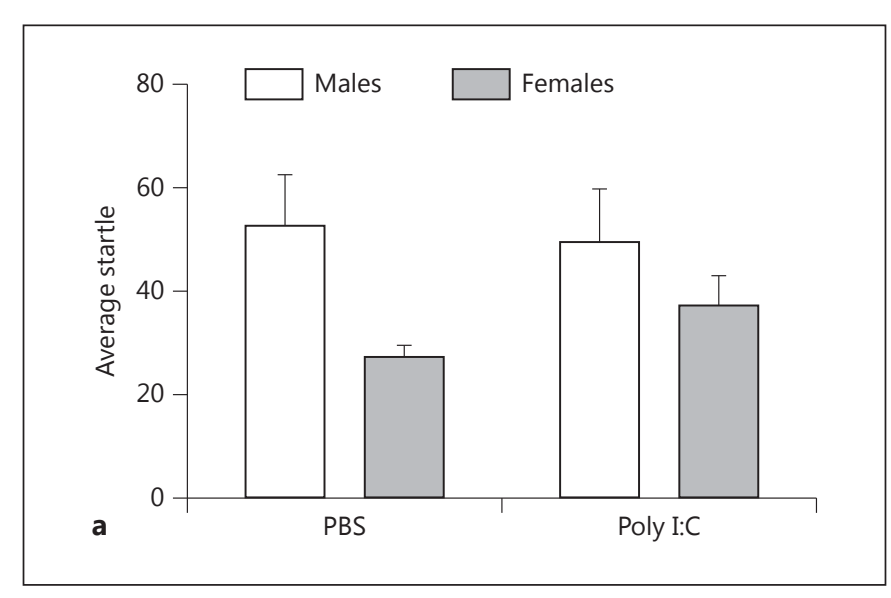

Fig. 6. Effects of prenatal exposure to PBS or poly I:C on average startle (a) as well as \%PPI for each prepulse intensity and average $\%$ PPI in male (b) and female (c) offspring. There was no difference in average startle between the treatment groups (a). Poly I:C animals showed a significant decrease in \%PPI for each prepulse intensity average \%PPI (b, $\mathbf{c} ; \mathrm{p}<0.01)$, irrespective of sex. ${ }^{*} \mathrm{p}<0.05$ compared with PBS animals. The data are shown as means \pm SEM in all graphs.

of memory and learning, when tested at 3-5 weeks of postnatal age, a stage of development that is equivalent to late childhood and prepubescence in humans [32]. Whereas previous studies have described behavioural abnormalities in adult animals after poly I:C treatment in pregnancy $[18,20,22]$, the emergence of symptoms in childhood and early adolescence is an important finding, since some mental illnesses such as autism, ADHD and schizophrenia, thought to be provoked by maternal viral illness during pregnancy, emerge at this stage of life. While the dose of poly I:C used in this study was higher than that used in our previous study [45], it is similar to that used in other studies with conventional mice or rats which produce altricial offspring. This poly I:C dose $(5 \mathrm{mg} / \mathrm{kg}$ ) had no effect on the duration of pregnancy, litter size and neonatal survival; however, unlike that used in our previous study, this higher dose of poly I:C did cause an acute decrease in maternal body weight, an indicator of sickness behaviour used in previous studies [66]. As prenatal poly I:C administration caused a decrease in postnatal growth, although not significantly, it

Behavioural Impact of Prenatal Immune Alteration
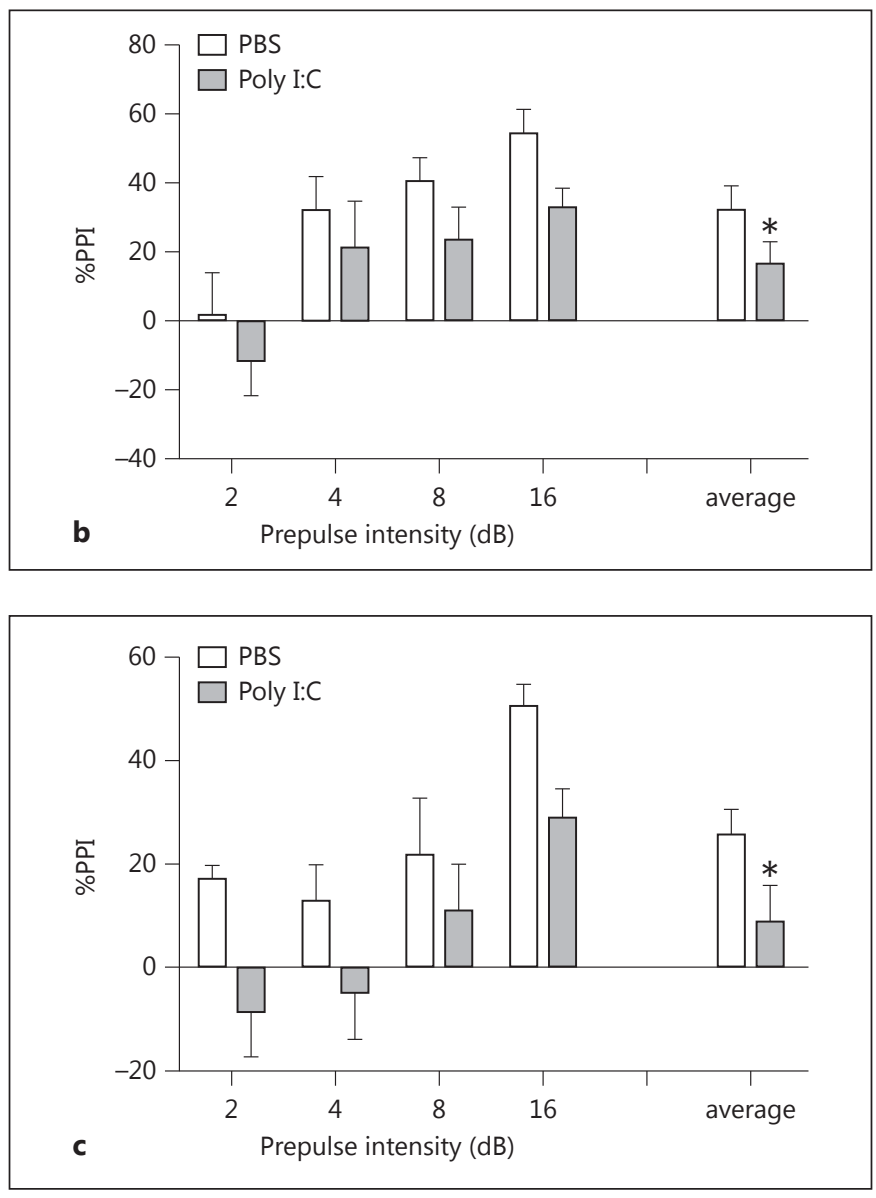

is important to consider whether this higher dose of poly I:C may have caused a global compromise and, if so, what effect this may have had on postnatal behaviour.

Our test of social interaction was based on social novelty and the natural curiosity of the animal to investigate and interact with a stranger spiny mouse. This test showed that male offspring born to mothers given poly I:C at mid gestation spent less time interacting with the stranger than controls, as indicated by the reduction in sniffing at the stranger's enclosure, a common method of social investigation for rodents [67]. The awareness of the presence of the stranger by the poly I:C-treated offspring appeared to be unaffected, as the control and poly I:C-treated animals spent a similar amount of time in the chamber containing the stranger spiny mouse. In addition, it is unlikely that the reduction in interaction has been affected by an increase in non-specific fear or anxiety, low curiosity or reduced exploratory activity, because the results from the elevated plus maze and the open field tests showed no difference in these behaviours between PBS and poly I:C animals. The social interaction test thus 
shows a resistance of the juvenile, male poly I:C offspring to come into close association with an unknown, 'stranger' individual. This result is of particular relevance to schizophrenia and autism, with the age at onset of schizophrenia being earlier in males than in females [68], and with autistic males more severely affected than female patients [69-71].

Abnormal social interaction including reduced interest in peers, difficulty in maintaining social interaction, and unusual modes of social interaction have been used as a diagnostic measure for identifying autism in humans [72], although only few animal studies have investigated the impact of poly I:C given during pregnancy on social behaviours of the offspring. This study shows that a single viral mimetic treatment at mid gestation has an impact that results in altered social behaviour in male offspring at an age that corresponds to the approximate time of puberty in this precocial species. Previous studies in mice showing effects on social behaviours differ in that either the dose of poly I:C was very high $(20 \mathrm{mg} / \mathrm{kg})[73,74]$ or repeated poly I:C treatments were given directly to mouse pups at 2-6 days of postnatal age [75]. Further studies on the social behaviour of these animals in the home cage environment, and in groups of other juvenile or adult spiny mice, could be important in establishing whether the results of this simple 'stranger' interaction test extend to more complex social interactions and settings.

PPI was used to assess sensorimotor gating, an index of the ability of the brain to filter and process incoming sensory information. A decrease in PPI is a common feature of neuropsychiatric disorders such as autism and schizophrenia [76-79], and changes in PPI have been used to screen potential antipsychotic drugs in human $[80,81]$ and animal studies $[82,83]$. In the current study we found a significant decrease in PPI in both male and female juvenile offspring born to mothers given poly I:C. This complements previous results in rats, where a reduced PPI has been observed in juvenile and adult offspring after prenatal poly I:C exposure [84], although other studies in juvenile and adult mice found a decreased PPI only in the adults [85]. Importantly, the assumption that deficits in PPI do not emerge until adulthood has not been consistently confirmed, and human patients identified as being at risk of schizophrenia clearly show that sensorimotor gating impairments may be evident prior to the full onset of the condition [86, 87]. Variation in developmental trajectories of the multiple brain regions and of the neurochemical substrates involved in regulating auditory PPI may explain the differences in the emergence of PPI deficits between species. Differences in PPI deficits and other behaviours between species may also be the result of the timing of prenatal poly I:C administration, as the stage of fetal development may be important for prompting changes in brain development that persist after birth.

Memory impairment is a well-documented symptom in mental illnesses such as schizophrenia and autism [8890], and a significant association between impaired cognition and both schizophrenia and autism have been identified in human patients [57, 91]. Our novel object recognition test conducted on the spiny mouse found that the poly I:C treatment during pregnancy impaired the capacity of 20-day-old male and female offspring to detect a novel object, compared with offspring from control pregnancies. A previous study in mice showed that prenatal treatment with poly I:C affected novel object recognition in adult offspring [75], and, again to our knowledge, this has not previously been tested or shown to occur at more juvenile ages.

In addition to non-spatial recognition memory, human studies have found impairments of working memory and reversal learning in schizophrenic $[88,92,93]$ and autistic $[89,94]$ patients. It would be of interest to assess working memory and reversal learning in a study like the present one to provide further validation of our model.

We have shown a significant behavioural impact arising in the juvenile offspring of spiny mice when the mother was given a dose of poly I:C that caused a downregulation of proinflammatory cytokines in the fetal brain. A future direction would be to determine whether behavioural abnormalities in offspring persist, intensify or attenuate in adulthood and old age. Nonetheless, we believe our findings of behavioural abnormalities in young animals are important as groups at risks of psychosis present with behavioural deficits prior to the onset of a disease $[86,87]$. Therefore, this period prior to puberty may provide a unique period of time for the development of interventions or treatments.

\section{Acknowledgements}

This research has been undertaken in the authors' capacity as a staff member, student or affiliate of the Monash Institute of Medical Research, Monash University. This study has been supported by funding from the National Health and Medical Research Council and the Victorian Government's Operational Infrastructure Support Program. U.R. and T.Q. were in receipt of an Australian Postgraduate Award scholarship during the period of this study. H.D. is funded by an ARC Australian Post-Doctoral Research Fellowship. D.W.W. was a National Health and Medical Research Council Principal Research Fellow and is a Senior Scientist at the Monash Institute of Medical Research.
Ratnayake/Quinn/LaRosa/Dickinson/ Walker 


\section{References}

$\checkmark 1$ Brown AS, et al: Maternal exposure to respiratory infections and adult schizophrenia spectrum disorders: a prospective birth cohort study. Schizophr Bull 2000;26:287-295.

2 Brown AS, et al: Nonaffective psychosis after prenatal exposure to rubella. Am J Psychiatry 2000;157:438-443.

- 3 Brown AS, et al: Serologic evidence of prenatal influenza in the etiology of schizophrenia. Arch Gen Psychiatry 2004;61:774-780.

4 Brown AS, et al: Maternal exposure to toxoplasmosis and risk of schizophrenia in adult offspring. Am J Psychiatry 2005;162:767-773.

5 Buka SLS, et al: Maternal infections and subsequent psychosis among offspring. Arch Gen Psychiatry 2001;58:1032-1037.

6 Fuller Torrey E, Rawlings R, Waldman IN: Schizophrenic births and viral diseases in two states. Schizophr Res 1988;1:73-77.

7 Mortensen PB, et al: Toxoplasma gondii as a risk factor for early-onset schizophrenia: analysis of filter paper blood samples obtained at birth. Biol Psychiatry 2007;61:688693.

8 Suvisaari J, et al: Association between prenatal exposure to poliovirus infection and adult schizophrenia. Am J Psychiatry 1999;156: 1100-1102.

9 Babulas V, et al: Prenatal exposure to maternal genital and reproductive infections and adult schizophrenia. Am J Psychiatry 2006; 163:927-929.

10 Watanabe Y, Someya T, Nawa H: Cytokine hypothesis of schizophrenia pathogenesis: evidence from human studies and animal models. Psychiatry Clin Neurosci 2010;64:217230.

11 Ashdown $\mathrm{H}$, et al: The role of cytokines in mediating effects of prenatal infection on the fetus: implications for schizophrenia. Mol Psychiatry 2006;11:47-55.

-12 Meyer U, Feldon J, Yee BK: A review of the fetal brain cytokine imbalance hypothesis of schizophrenia. Schizophr Bull 2009;35:959972.

13 Gilmore J, Jarskog LF: Exposure to infection and brain development: cytokines in the pathogenesis of schizophrenia. Schizophr Res 1997;24:365-367.

14 Cunningham C, et al: The sickness behaviour and CNS inflammatory mediator profile induced by systemic challenge of mice with synthetic double-stranded RNA (poly I:C). Brain Behav Immun 2007;21:490-502.

15 Gandhi R, et al: Influence of poly I:C on sickness behaviors, plasma cytokines, corticosterone and central monoamine activity: moderation by social stressors. Brain Behav Immun 2007;21:477-489.

16 Fortier ME, et al: The viral mimic, polyinosinic:polycytidylic acid, induces fever in rats via an interleukin-1-dependent mechanism. Am J Physiol Regul Integr Comp Physiol 2004;287:R759-R766.
17 Zuckerman L, et al: Immune activation during pregnancy in rats leads to a postpubertal emergence of disrupted latent inhibition, dopaminergic hyperfunction, and altered limbic morphology in the offspring: a novel neurodevelopmental model of schizophrenia. Neuropsychopharmacology 2003;28:1778-1789.

18 Meyer U, et al: Towards an immuno-precipitated neurodevelopmental animal model of schizophrenia. Neurosci Biobehav Rev 2005; 29:913-947.

19 Zuckerman L, Weiner I: Maternal immune activation leads to behavioral and pharmacological changes in the adult offspring. J Psychiatr Res 2005;39:311-323.

20 Ozawa KK, et al: Immune activation during pregnancy in mice leads to dopaminergic hyperfunction and cognitive impairment in the offspring: a neurodevelopmental animal model of schizophrenia. Biol Psychiatry 2006; 59:546-554.

21 Wolff AR, Bilkey DK: Immune activation during mid-gestation disrupts sensorimotor gating in rat offspring. Behav Brain Res 2008; 190:156-159.

22 Makinodan M, et al: Maternal immune activation in mice delays myelination and axonal development in the hippocampus of the offspring. J Neurosci Res 2008;86:2190-2200.

23 Howland JG, Cazakoff BN, Zhang Y: Altered object-in-place recognition memory, prepulse inhibition, and locomotor activity in the offspring of rats exposed to a viral mimetic during pregnancy. Neuroscience 2012;201: 184-198.

24 Schwendener S, Meyer U, Feldon J: Deficient maternal care resulting from immunological stress during pregnancy is associated with a sex-dependent enhancement of conditioned fear in the offspring. J Neurodev Disord 2009; $1: 15-32$.

25 de Miranda J, et al: Induction of Toll-like receptor 3-mediated immunity during gestation inhibits cortical neurogenesis and causes behavioral disturbances. MBio 2010; e00176-10.

26 Clancy B, Darlington RB, Finlay BL: Translating developmental time across mammalian species. Neuroscience 2001;105:7-17.

27 Dickinson H, et al: The spiny mouse (Acomys cahirinus) completes nephrogenesis before birth. Am J Physiol Renal Physiol 2005; 289:F273-F279.

28 Oosterhuis WWP, et al: Perinatal development of the lung in rat and spiny mouse: its relation to altricial and precocial timing of birth. Biol Neonate 1984;45:236-243.

29 Lamers WH, et al: Perinatal development of the liver in rat and spiny mouse: its relation to altricial and precocial timing of birth. Eur J Biochem 1985;146:475-480.

30 Brunjes PC, Korol DL, Stern KG: Prenatal neurogenesis in the telencephalon of the precocial mouse Acomys cahirinus. Neurosci Lett 1989;107:114-119.
31 Brunjes PC: A stereological study of neocortical maturation in the precocial mouse, Acomys cahirinus. Brain Res 1985;351:279-287.

32 Quinn TA, et al: Ontogeny of the adrenal gland in the spiny mouse, with particular reference to production of the steroids cortisol and dehydroepiandrosterone. Endocrinology 2013;154:1190-1201.

33 Takeda K, Akira S: Toll-like receptors in innate immunity. Int Immunol 2005;17:1-14.

34 Alexopoulou L, et al: Recognition of doublestranded RNA and activation of NF- $\kappa B$ by Toll-like receptor 3. Nature 2001;413:732738.

35 Koga K, Mor G: Expression and function of toll-like receptors at the maternal-fetal interface. Reprod Sci 2008;15:231-242.

36 Takeuchi O, Akira S: Recognition of viruses by innate immunity. Immunol Rev 2007;220: 214-224.

37 Ashwood P, et al: Elevated plasma cytokines in autism spectrum disorders provide evidence of immune dysfunction and are associated with impaired behavioral outcome. Brain Behav Immun 2011;25:40-45.

38 Chez MG, et al: Elevation of tumor necrosis factor- $\alpha$ in cerebrospinal fluid of autistic children. Pediatr Neurol 2007;36:361-365.

39 Maes M, et al: Increased serum interleukin-8 and interleukin-10 in schizophrenic patients resistant to treatment with neuroleptics and the stimulatory effects of clozapine on serum leukemia inhibitory factor receptor. Schizophr Res 2002;54:281-291.

40 Potvin S, et al: Inflammatory cytokine alterations in schizophrenia: a systematic quantitative review. Biol Psychiatry 2008;63:801-808.

41 Arrode-Bruses G, Bruses JL: Maternal immune activation by poly(I:C) induces expression of cytokines IL- $1 \beta$ and IL-13, chemokine MCP-1 and colony stimulating factor VEGF in fetal mouse brain. J Neuroinflammation 2012;9:83.

42 Meyer U, et al: Immunological stress at the maternal-foetal interface: a link between neurodevelopment and adult psychopathology. Brain Behav Immun 2006;20:378-388.

43 Meyer U, et al: The time of prenatal immune challenge determines the specificity of inflammation-mediated brain and behavioral pathology. J Neurosci 2006;26:4752-4762.

44 Gilmore JH, Jarskog LF, Vadlamudi S: Maternal poly I:C exposure during pregnancy regulates TNFa, BDNF, and NGF expression in neonatal brain and the maternal-fetal unit of the rat. J Neuroimmunol 2005;159:106-112.

45 Ratnayake U, et al: Behaviour and hippocampus-specific changes in spiny mouse neonates after treatment of the mother with the viralmimetic poly I:C at mid-pregnancy. Brain Behav Immun 2012;26:1288-1299.

46 Ito HT, et al: Maternal immune activation alters nonspatial information processing in the hippocampus of the adult offspring. Brain Behav Immun 2010;24:930-941. 
$\checkmark 47$ Meyer U, et al: Adult brain and behavioral pathological markers of prenatal immune challenge during early/middle and late fetal development in mice. Brain Behav Immun 2008;22:469-486.

-48 Li Q, et al: Prenatal immune challenge is an environmental risk factor for brain and behavior change relevant to schizophrenia: evidence from MRI in a mouse model. PLoS One 2009;4:e6354.

49 Dickinson H, et al: A comparative study of renal function in the desert-adapted spiny mouse and the laboratory-adapted C57BL/6 mouse: response to dietary salt load. Am J Physiol Renal Physiol 2007;293:F1093F1098.

50 Dickinson H, Walker DW: Managing a colony of spiny mice (Acomys cahirinus) for perinatal research. ANZCCART News 2007;20: 4-11.

51 Pietz B: The oestrous cycle of the spiny mouse (Acomys cahirinus). J Reprod Fertil 1981;61: 453-459.

52 Ireland Z, et al: Developmental changes in the expression of creatine synthesizing enzymes and creatine transporter in a precocial rodent, the spiny mouse. BMC Dev Biol 2009;9:39.

53 Benveniste EN: Cytokine actions in the central nervous system. Cytokine Growth Factor Rev 1998;9:259-275.

54 Deverman BE, Patterson PH: Cytokines and CNS development. Neuron 2009;64:61-78.

55 Jonakait GM: Cytokines in neuronal development. Adv Pharmacol 1997;37:35-67.

56 Jokhi PP, King A, Loke YW: Cytokine production and cytokine receptor expression by cells of the human first trimester placentaluterine interface. Cytokine 1997;9:126-137.

-57 Paradowska E, Blach-Olszewska Z, Gejdel E: Constitutive and induced cytokine production by human placenta and amniotic membrane at term. Placenta 1997;18:441-446.

- 58 Silverman MN, et al: Immune modulation of the hypothalamic-pituitary-adrenal (HPA) axis during viral infection. Viral Immunol 2005; 18:41-78.

59 Chrousos GP, Kino T: Ikaros transcription factors: flying between stress and inflammation. J Clin Invest 2005;115:844-848.

60 Chrousos GP: The hypothalamic-pituitaryadrenal axis and immune-mediated inflammation. N Engl J Med 1995;332:1351-1362.

61 Lee YB, Nagai A, Kim SU: Cytokines, chemokines, and cytokine receptors in human microglia. J Neurosci Res 2002;69:94-103.

62 Giulian D, et al: Interleukin-1 is an astroglial growth factor in the developing brain. J Neurosci 1988;8:709-714.
63 Rezaie P, et al: Expression of $\beta$-chemokines and chemokine receptors in human fetal astrocyte and microglial co-cultures: potential role of chemokines in the developing CNS. Glia 2002;37:64-75.

64 Hua LL, Lee SC: Distinct patterns of stimulusinducible chemokine mRNA accumulation in human fetal astrocytes and microglia. Glia 2000;30:74-81.

65 Hanisch UK: Microglia as a source and target of cytokines. Glia 2002;40:140-155.

66 Gibney SM, et al: Poly I:C-induced activation of the immune response is accompanied by depression and anxiety-like behaviours, kynurenine pathway activation and reduced BDNF expression. Brain Behav Immun 2013; 28:170-181.

67 Page DT, Kuti OJ, Sur M: Computerized assessment of social approach behavior in mouse. Front Behav Neurosci 2009;3:48.

68 Loranger AW: Sex difference in age at onset of schizophrenia. Arch Gen Psychiatry 1984; 41:157-161.

69 McLennan JD, Lord C, Schopler E: Sex differences in higher functioning people with autism. J Autism Dev Disord 1993;23:217227.

70 Lord C, Schopler E, Revicki D: Sex differences in autism. J Autism Dev Disord 1982;12:317330.

71 le Couteur A, et al: Autism diagnostic interview: a standardized investigator-based instrument. J Autism Dev Disord 1989;19:363387.

72 Silverman JL, et al: Behavioural phenotyping assays for mouse models of autism. Nat Rev Neurosci 2010;11:490-502.

73 Shi L, et al: Activation of the maternal immune system alters cerebellar development in the offspring. Brain Behav Immun 2009;23: 116-123.

74 Smith SEP, et al: Maternal immune activation alters fetal brain development through interleukin-6. J Neurosci 2007;27:10695-10702.

75 Ibi D, et al: Neonatal poly I:C treatment in mice results in schizophrenia-like behavioral and neurochemical abnormalities in adulthood. Neurosci Res 2009;64:297-305.

76 Grillon C, et al: Startle gating deficits occur across prepulse intensities in schizophrenic patients. Biol Psychiatry 1992;32:939-943.

77 Perry W, Geyer MA, Braff DL: Sensorimotor gating and thought disturbance measured in close temporal proximity in schizophrenic patients. Arch Gen Psychiatry 1999;56:277281.

78 Perry W, et al: Sensorimotor gating deficits in bipolar disorder patients with acute psychotic mania. Biol Psychiatry 2001;50:418-424.

79 Perry W, et al: Sensorimotor gating deficits in adults with autism. Biol Psychiatry 2007;61: 482-486.
80 Kumari V, Soni W, Sharma T: Normalization of information processing deficits in schizophrenia with clozapine. Am J Psychiatry 1999; 156:1046-1051.

81 Weike AI, Bauer U, Hamm AO: Effective neuroleptic medication removes prepulse inhibition deficits in schizophrenia patients. Biol Psychiatry 2000;47:61-70.

82 Shi L, et al: Maternal influenza infection causes marked behavioral and pharmacological changes in the offspring. J Neurosci 2003; 23:297-302.

83 le Pen G, Moreau JL: Disruption of prepulse inhibition of startle reflex in a neurodevelopmental model of schizophrenia: reversal by clozapine, olanzapine and risperidone but not by haloperidol. Neuropsychopharmacology 2002;27:1-11.

84 Wolff AR, Bilkey DK: The maternal immune activation (MIA) model of schizophrenia produces pre-pulse inhibition (PPI) deficits in both juvenile and adult rats but these effects are not associated with maternal weight loss. Behav Brain Res 2010;213:323-327.

85 Vuillermot $S$, et al: A longitudinal examination of the neurodevelopmental impact of prenatal immune activation in mice reveals primary defects in dopaminergic development relevant to schizophrenia. J Neurosci 2010;30:1270-1287.

86 Schell AM, et al: Attentional modulation of startle in psychosis-prone college students. Psychophysiology 1995;32:266-273.

87 Myles-Worsley M, et al: P50 sensory gating in adolescents from a pacific island isolate with elevated risk for schizophrenia. Biol Psychiatry 2004;55:663-667.

88 Lee J, Park S: Working memory impairments in schizophrenia: a meta-analysis. J Abnorm Psychol 2005;114:599-611.

89 Bennetto L, Pennington BF, Rogers SJ: Intact and impaired memory functions in autism. Child Dev 1996;67:1816-1835.

90 Minshew NJ, Goldstein G: The pattern of intact and impaired memory functions in autism. J Child Psychol Psychiatry 2001;42: 1095-1101.

91 Aleman A, et al: Memory impairment in schizophrenia: a meta-analysis. Am J Psychiatry 1999;156:1358-1366.

92 Saykin AJ, et al: Neuropsychological function in schizophrenia: selective impairment in memory and learning. Arch Gen Psychiatry 1991;48:618-624.

93 Waltz JA, Gold JM: Probabilistic reversal learning impairments in schizophrenia: further evidence of orbitofrontal dysfunction. Schizophr Res 2007;93:296-303.

94 Lionello-Denolf KM, et al: Reversal learning set and functional equivalence in children with and without autism. Psychol Rec 2008; 58:15-36. 